\title{
Plasmonic Coupler and Multiplexer/Demultiplexer Based on Nano-Groove-Arrays
}

\author{
Aparna Udupi ${ }^{1} \cdot$ Sathish Kumar Madhava ${ }^{1}$ (D)
}

Received: 28 December 2020 / Accepted: 15 March 2021 / Published online: 26 March 2021

(c) The Author(s) 2021

\begin{abstract}
A novel plasmonic unidirectional coupler and its extension to a multiplexer/demultiplexer are proposed and simulated. The proposed structure can be etched adjacent to metal-insulator-metal (MIM) waveguides which can result in considerable reduction in footprint of optical signal processing systems. Simulated results show very good agreement with design. Extinction ratio (ER) of more than $11 \mathrm{~dB}$ was achieved for both the coupler as well as the multiplexer/demultiplexer. Crosstalk as well as full width at half maximum (FWHM) was also highly acceptable for the multiplexer/demultiplexer. The proposed structures have an additional attractive feature of being amenable to integration with other relevant functionalities as demonstrated in the results.
\end{abstract}

Keywords Plasmonics $\cdot$ Surface plasmon polariton $\cdot$ MIM devices $\cdot$ Coupler $\cdot$ Multiplexer $\cdot$ Demultiplexer

\section{Introduction}

Surface plasmon polariton (SPP) is collective oscillations of free electrons on the metal surface that are coupled to an external electromagnetic field, propagating along a metaldielectric interface [1]. Because of their ability to break the diffraction limit, strongly enhance, and manipulate light at sub-wavelength scales, SPPs are believed to be promising candidates for constructing the next-generation ultra-compact integrated photonic circuits for information processing [2, 3]. Plasmonic waveguides, which primarily work on the principle of SPP waves, have been very popular for the construction of extremely compact photonic devices $[2,4]$. Metal-insulator-metal (MIM) waveguides, which consist of a dielectric core and two metallic cladding layers, have drawn more attention due to the strong localization of SPP modes which they can provide over a very wide range of wavelengths with an acceptable propagation length and ease of fabrication [3]. Consequently, MIM waveguides have been used widely for applications such as sensors, logic gates, filters, lenses, and switches [5-10].

Sathish Kumar Madhava

sathish.kumar@manipal.edu

1 Department of Electronics and Communication Engineering, Manipal Institute of Technology, Manipal Academy of Higher Education, Manipal, Karnataka, India
Conversion of free-space light waves impinging on a metal surface into SPP waves and further guiding those SPP waves in desired directions through an MIM waveguide has always been a challenge due to the mismatch in wave vectors: $\boldsymbol{k}_{\mathbf{0}}$ and $\boldsymbol{k}_{\text {spp }}$. Plasmonic couplers, which can perform the task of coupling free space light waves onto MIM waveguides, have hence attracted a lot of research attention. Towards this, different techniques such as grooves, slits, or gratings placed appropriately have been adopted by many researchers [11-15].

Considering the fact that plasmonics enabled optical information processing systems will have to operate on more than one wavelength, investigations on compact devices to multiplex and demultiplex wavelengths have become a major research topic as evidenced in recent published literature. MIM waveguides in conjunction with different types of resonators have been proposed to act as a multiplexer and demultiplexer [16-19]. Other than the resonators, techniques such as multimode interference effect, integration of different periodic gratings, and multiple-wavelength focusing and demultiplexing have also been investigated in [20-25] and a few of them have also been fabricated [20, 21].

In this paper, a nano-groove-array-based unidirectional coupler is proposed which couples SPP waves from one MIM waveguide to another placed parallel to it. Depending on the directions of coupling, different cases have been considered and effect of groove spacing in the array has been 
discussed. Further by exploiting the versatility of the nanogroove-array, a $1 \times 2$ multiplexer/demultiplexer is explored. Considering the importance of compact integrated photonic circuits with as small a footprint as possible, the proposed structure is of high relevance since by nature, this structure can be integrated along with other signal processing structures on MIM waveguides.

\section{Design}

The schematic of the proposed two-dimensional coupler is as shown in Fig. 1. The width of both MIM waveguides as well as the grooves are adjusted to be such that only the fundamental $\left(\mathrm{TM}_{0}\right)$ SPP mode is supported by them for the range of wavelength of operation. MIM waveguide at the top is referred as the bus and that at the bottom, parallel to the bus, as the coupling waveguide. SPP wave propagating in the bus couples to the rectangular nano-groove-array evanescently and each groove emits light like a point source. Through a proper choice of the spacing of the individual grooves in the array, the emissions from the point sources can be constructively added to obtain a plane wave a few nanometers away from the nano-groove-array and which propagates in a chosen direction [26]. The emitted plane wave can be coupled into the coupling MIM waveguide in the desired direction, placed at a distance $d$, by etching a matching nano-groove-array on it. It may be noted that the progressive variation in the groove depths in the waveguides is to ensure uniform emission and coupling of power [26]. Needless to say, by virtue of Helmholtz reciprocity theorem [27], the roles of the bus and coupling waveguides can be reversed in that SPP waves propagating in the lower waveguide can be coupled on to the waveguide at the top. The schematic would look exactly like the one in Fig. 1 except for the fact that the directions of all the arrows indicating propagation directions will reverse.

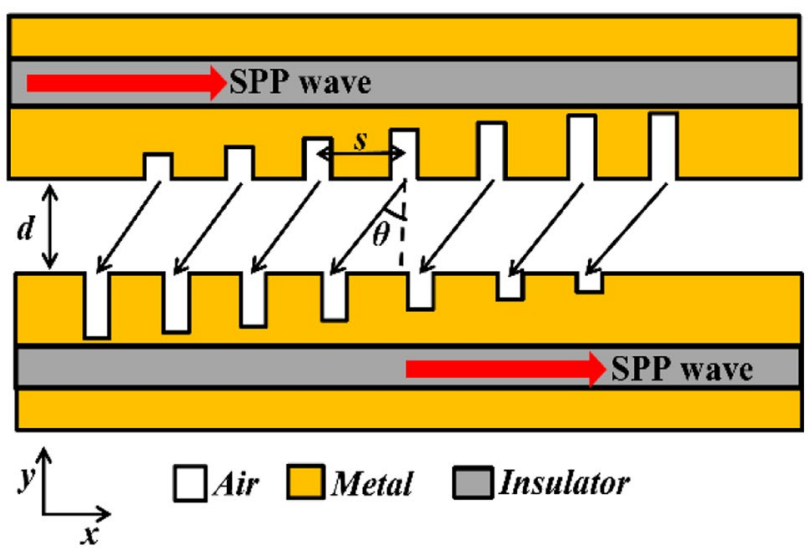

Fig. 1 Schematic of the proposed unidirectional coupler
For SPP wave in the bus to propagate as a plane wave in free space in a desired direction, position of individual grooves in the array must be adjusted so that the field emitted by them add constructively in the desired direction. This spacing between the grooves $s$ can be arrived at from the expression given in [26]. The expression in [26] can be further written in a more elegant form as discussed here. The $\beta$ and $k_{0}$ in [26] stand, respectively, for the magnitudes of propagation constant of SPP, also identified as $k_{\text {spp }}$, and the free space propagation constant. With $\sin (\theta)$ expressed as $-\cos (\theta+\pi / 2)$ and using the definition of dot product between vectors, the equation in [26] becomes

$s=\frac{2 n \pi}{\left(\boldsymbol{k}_{s p p}-\boldsymbol{k}_{0}\right) \cdot \boldsymbol{a}_{k s p}}$

where as mentioned above, $\boldsymbol{k}_{\boldsymbol{s p}}$ is the SPP wave vector which can be calculated as explained in [1], $n$ is a non-zero positive integer, $\boldsymbol{k}_{\mathbf{0}}$ is the free-space wave vector, and $\boldsymbol{a}_{\boldsymbol{k} p p}$ is a unit vector along the direction of propagation of the SPP wave in the bus.

To ensure that the field which exited the bus through the nano-groove-array to be picked up and guided in the desired direction, a matching nano-groove-array has to be interfaced between free space and the coupling waveguide. The matching nano-groove-array must facilitate the constructive interfere of each of the SPP waves excited in the nano-grooves in the desired direction within the coupling waveguide [28]. This suggests that, by virtue of Helmholtz reciprocity theorem, the matching nano-groove-array structure can also be arrived at using (1). It should be noted that sufficient number of grooves are required in both the arrays to obtain a highly efficient coupling.

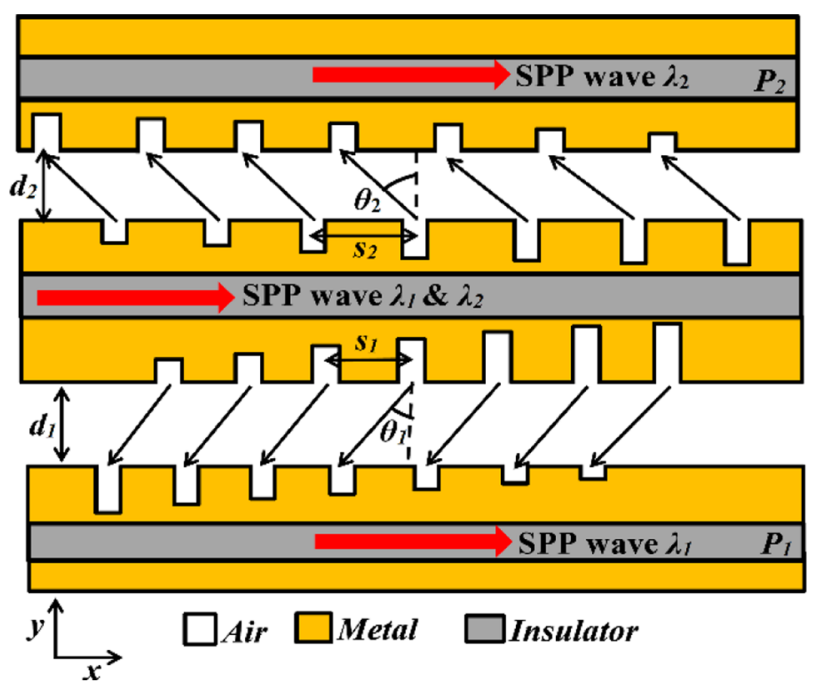

Fig. 2 Schematic of the proposed multiplexer/demultiplexer 

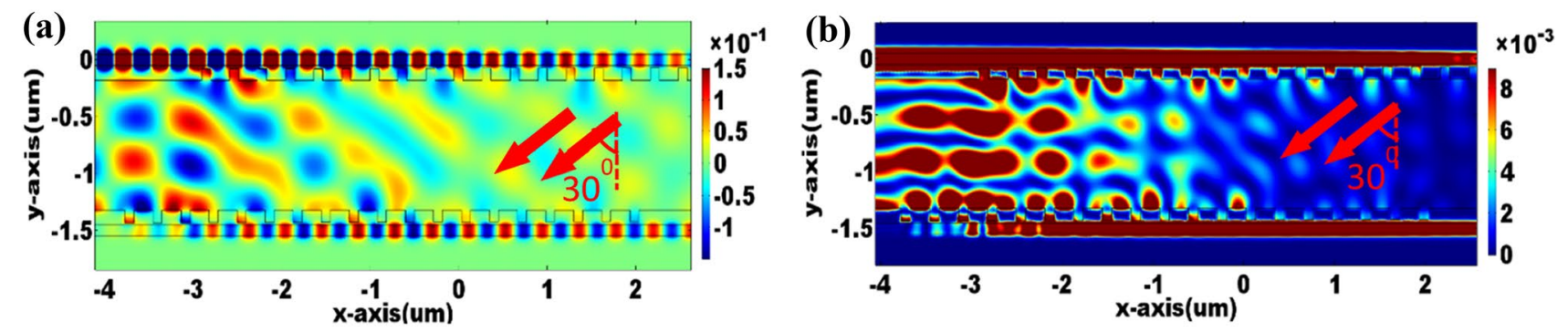

Fig. 3 Magnetic field $\left(H_{z}\right)$ in (a) and its intensity $\left(\left|H_{z}\right|^{2}\right)$ in (b) for the proposed plasmonic coupler designed for case 1

As mentioned earlier, for uniform emission and coupling of power, the metal-barrier thickness between the core and the nano-groove-array of the waveguides has to vary progressively. Due to the exponential decay of power within waveguides [1], this variation $t_{\mathrm{m}}$ has to be exponential and is as given below [26].

$t_{\mathrm{m}}=A \exp (-a s(m+p))$

Here, $A$ and $a$ are the constants, $m$ is zero for the groove at the center of the array and takes on positive integer values for nano-grooves on the right and negative integer values for nano-grooves on the left while $p=\max (|m|)$.

It can be noted that a properly designed nano-groovearray can emit a highly directional beam for a particular wavelength. This can be readily observed from the fact that (1) is actually function of wavelength as reflected by the presence of $\boldsymbol{k}_{\boldsymbol{s p} p}$ and $\boldsymbol{k}_{\mathbf{0}}$ in it. Meanwhile, such an array of grooves can also suppress other wavelengths due to emissions from individual grooves in the array being not in phase. This feature is exploited to convert the above unidirectional coupler into a $1 \times 2$ multiplexer/demultiplexer.

The functioning of the $1 \times 2$ multiplexer/demultiplexer can be understood from Fig. 2. As can be seen from this figure, on either sides of the bus waveguide, nano-groove-arrays are etched. The array at the bottom is designed to evanescently pick up SPP wave of wavelength $\lambda_{1}$ and emit it as a plane wave propagating in a chosen direction defined by angle $\theta_{1}$ and the array at the top is designed to select a different wavelength $\lambda_{2}$ and emit it as a plane wave propagating in a direction defined by angle $\theta_{2}$.
As shown in this figure, the structure has two MIM waveguides with matching-nano-groove arrays etched on them placed parallel to the bus at distances $d_{1}$ and $d_{2}$, respectively. The array at the bottom waveguide is matched to couple the field of wavelength $\lambda_{1}$ and propagate it as an SPP wave in the desired direction. Similarly, the array at the top waveguide is designed to function in the same way for fields of wavelength $\lambda_{2} . P_{1}$ and $P_{2}$ have been identified as the output ports and are as shown in Fig. 2.

With the arrangement as explained above, when an SPP wave constituted of wavelengths $\lambda_{1}$ and $\lambda_{2}$ propagates through the bus and encounters the nano-groove-arrays, it gets separated and coupled into the respective waveguides for $\lambda_{1}$ and $\lambda_{2}$ kept adjacent to the bus, which completes the demultiplexing action. With the help of Helmholtz reciprocity theorem, it can be easily understood how this same arrangement works as a multiplexer. SPP wave of wavelength $\lambda_{1}$ propagating in the bottommost waveguide can couple into the bus and similarly SPP wave of wavelength $\lambda_{2}$ propagating in the topmost waveguide can couple into the bus. The schematic would look exactly like the one in Fig. 2 except for the fact that the directions of all the arrows indicating propagation directions will reverse.

\section{Results and Discussion}

The proposed two dimensional structures were simulated using COMSOL Multiphysics. All the involved MIM waveguide metal is modelled as Au with its complex permittivity
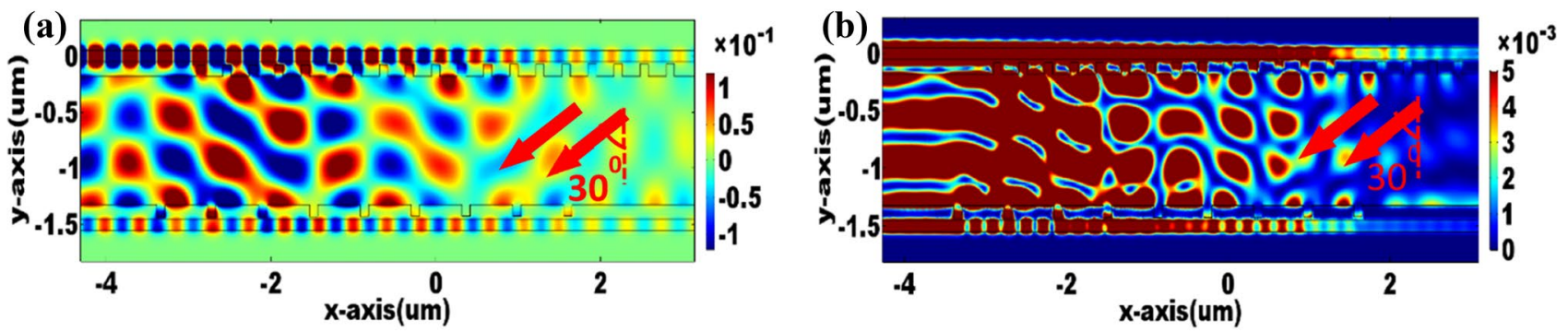

Fig. 4 Magnetic field $\left(H_{z}\right)(\mathbf{a})$ and its intensity $\left(\left|H_{z}\right|^{2}\right)(\mathbf{b})$ for the proposed plasmonic coupler designed for case 2 

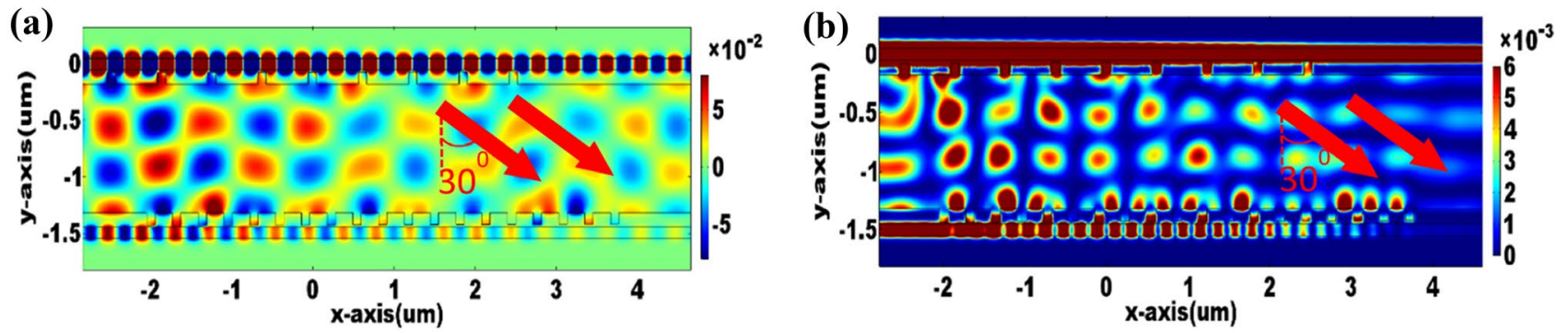

Fig. 5 Magnetic field $\left(H_{z}\right)$ in (a) and its intensity $\left(\left|H_{z}\right|^{2}\right)$ in $(\mathbf{b})$ for the proposed plasmonic coupler for modified case 2

obtained using the Drude model [29]. Dielectric material of the MIM waveguides is assumed to be $\mathrm{SiO}_{2}$ with refractive index 1.2. The widths of all the MIM waveguides in the structure as well as the width of nano-grooves in the array were set as $100 \mathrm{~nm}$. Through trial and error, $A=30 \mathrm{~nm}$ and $a=90 \times 10^{3}$ was arrived at for determining the variation in the metal-barrier thickness. The depth of the left (right) most groove on the bus (coupling) waveguide was fixed at $100 \mathrm{~nm}$ and the depth of the subsequent grooves increased progressively as per (2).

For demonstration of the functioning of the proposed structure as a coupler, two cases were considered as follows. In the first case, i.e., case 1 , direction of propagation of the SPP waves in the bus as well as the coupling waveguide is the same, and in the second case, i.e., case 2, these two directions are opposite to each other. For both the cases, operating wavelength is chosen as $650 \mathrm{~nm}$. The coupling waveguide was placed such that $d=1.5 \mu \mathrm{m}$. The size of the nano-groove-array was fixed at $6 \mu \mathrm{m}$.

To begin with, the nano-groove-array is designed on the bus to emit plane waves propagating in a direction such that $\boldsymbol{k}_{\mathbf{0}}$ defines an angle of $\theta=30^{\circ}$ to the left with respect to the normal to the bus as in Fig. 1. For case 1, number of grooves within the chosen array size of $6 \mu \mathrm{m}$ was found to be 19 with a groove spacing $s$ of $317 \mathrm{~nm}$ for the nano-groove-array on the bus as well as coupling waveguides. Figure $3 \mathrm{a}$, b show the plots of the $z$ component of the magnetic field $\left(H_{Z}\right)$ and its intensity $\left(\left|H_{Z}\right|^{2}\right)$ respectively. It can be clearly seen from the figure that SPP wave propagating in the bus is successfully converted first into a plane wave and then coupled into
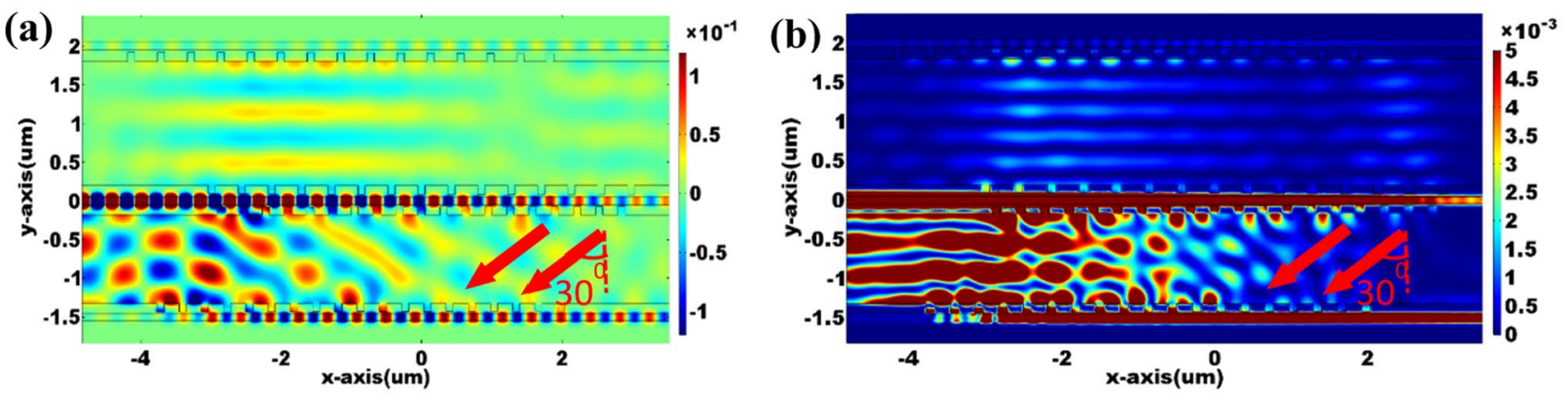

(c)
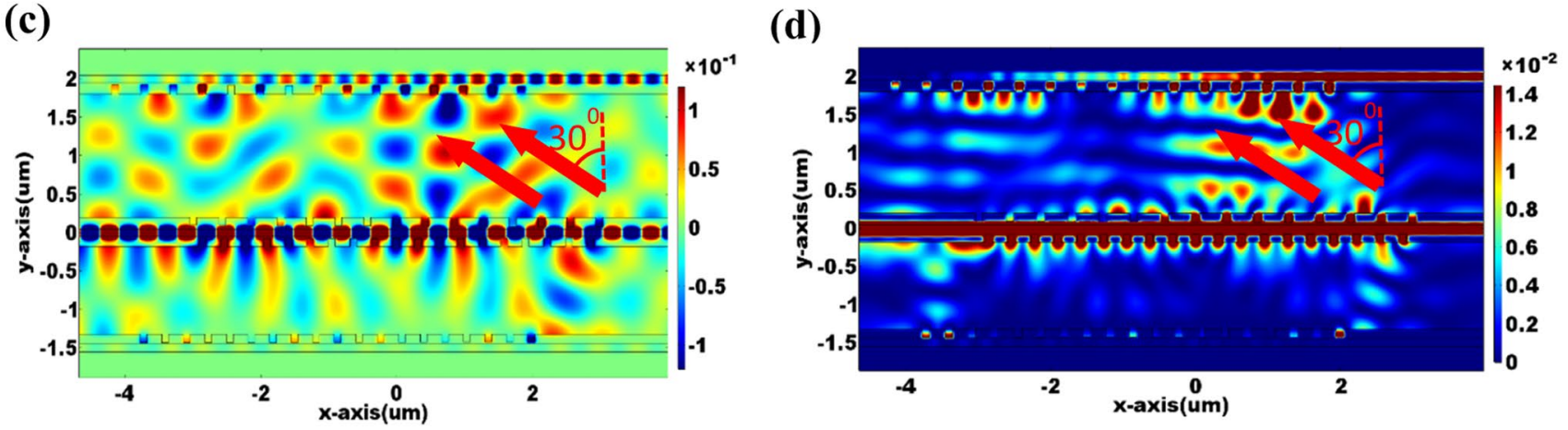

Fig. 6 Magnetic field $\left(H_{z}\right)$ in (a), (c) and its intensity $\left(\left|H_{z}\right|^{2}\right)$ in $(\mathbf{b}),(\mathbf{d})$ for the proposed demultiplexer with $650 \mathrm{~nm}$ and $850 \mathrm{~nm}$ wavelength respectively 


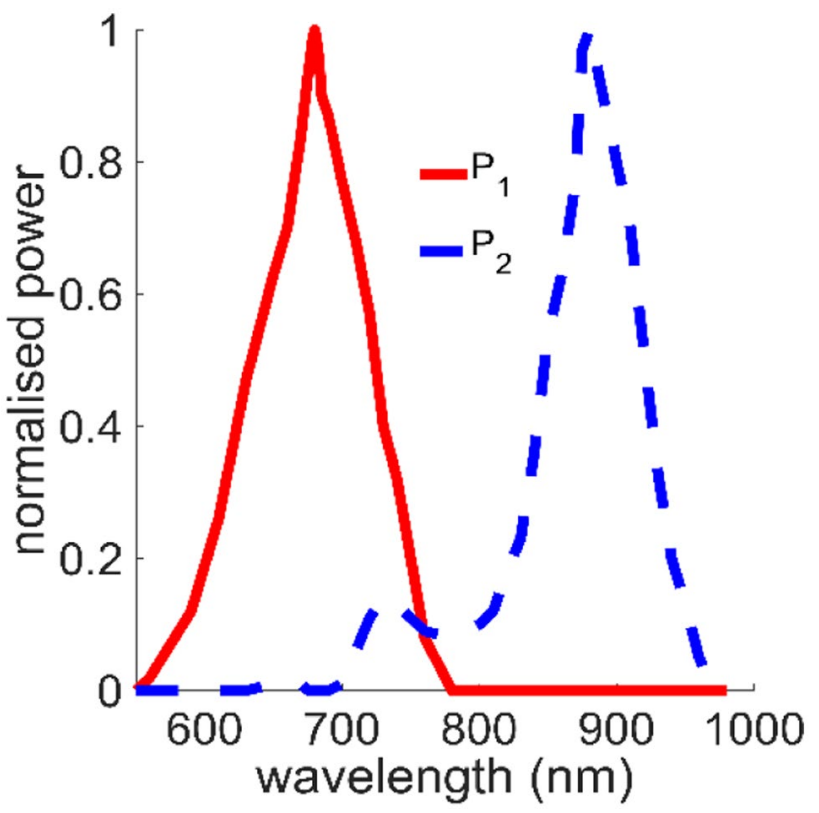

Fig. 7 Plot of time averaged normalized output power $\left(\left|P_{x}\right|\right)$ measured at ports $P_{1}$ and $P_{2}$ with respect to wavelength

the adjacent waveguide in the desired direction as per case 1 with very minimal energy flow in the opposite direction.

For case 2, while the nano-groove-array was unchanged on the bus waveguide, number of grooves within the chosen array size of $6 \mu \mathrm{m}$ was found to be 9 with a groove spacing $s$ of $618 \mathrm{~nm}$ for the nano-groove-array on the coupling waveguide. Figure $4 a, b$ show results for case 2 and it can be seen that the coupled SPP wave propagates in the opposite direction as desired with minimal power flow in the undesired direction. The increased distance between grooves on the coupling bus side has an impact on the intensity of constructive interference between the waves picked up by the individual grooves which are visible in the figure. To put it in numbers, a measure of time averaged $x$ component of power $\left(\left|P_{\mathrm{x}}\right|\right)$ in the desired direction in the coupling waveguide in case 2 was found to be $33 \%$ less than that in case 1 .
Consequently, extinction ratio (ER), defined as ratio between powers transmitted in the desired direction to that in the opposite direction, turns out to be $11 \mathrm{~dB}$ for case 2 while it is $16 \mathrm{~dB}$ for case 1 .

To improve the ER in case 2, the nano-groove-array on the bus is redesigned such that $\boldsymbol{k}_{\mathbf{0}}$ defines an angle of $\theta=30^{\circ}$ to the right instead of left with respect to the normal to the bus. From (1), it can be seen that at the coupling waveguide, this will bring down the spacing between the nano-grooves to $317 \mathrm{~nm}$ from $618 \mathrm{~nm}$ which will further result in an increase in number of nano-grooves from 9 to 19 within the array size of $6 \mu \mathrm{m}$. Results for case 2 with $\boldsymbol{k}_{\mathbf{0}}$ direction modified as above are shown in Fig. 5. In terms of ER, this modification resulted in an improvement from 11 to $15 \mathrm{~dB}$ due to the reason that decreased spacing between the nano-grooves resulted in an improved constructive interference in the desired direction in association with an increased destructive interference in the opposite direction.

It must, however, be noted that this change in direction of $\boldsymbol{k}_{\mathbf{0}}$ will result in a lesser number of nano-grooves at the bus waveguide. To be precise, it will drop from 19 to 9 as can be seen from a comparison of Figs. 4 and 5. This will result in a lesser amount of power drop from the bus waveguide. However, with more number of nano-grooves at the coupling waveguide side, total power coupled into the coupling waveguide will remain almost the same as it was earlier for case 2 .

It is also worth mentioning here that case 1 with $\boldsymbol{k}_{\mathbf{0}}$ defining an angle $\theta$ to the right instead of left with respect to the normal to the bus will result in increased spacing between the nano-grooves at both the waveguides. The consequent decrease in number of nano-grooves at both the waveguides for a given array size will result not only in a poorer ER but also in a reduced power coupling. Hence, we have not included this result here.

From the above discussions, it can be concluded that for case 1 type of coupling, the nano-groove-array should be such that $\boldsymbol{k}_{\mathbf{0}}$ defines an angle $\theta$ to the left with respect to the normal to the bus, and for case 2 type of coupling, the
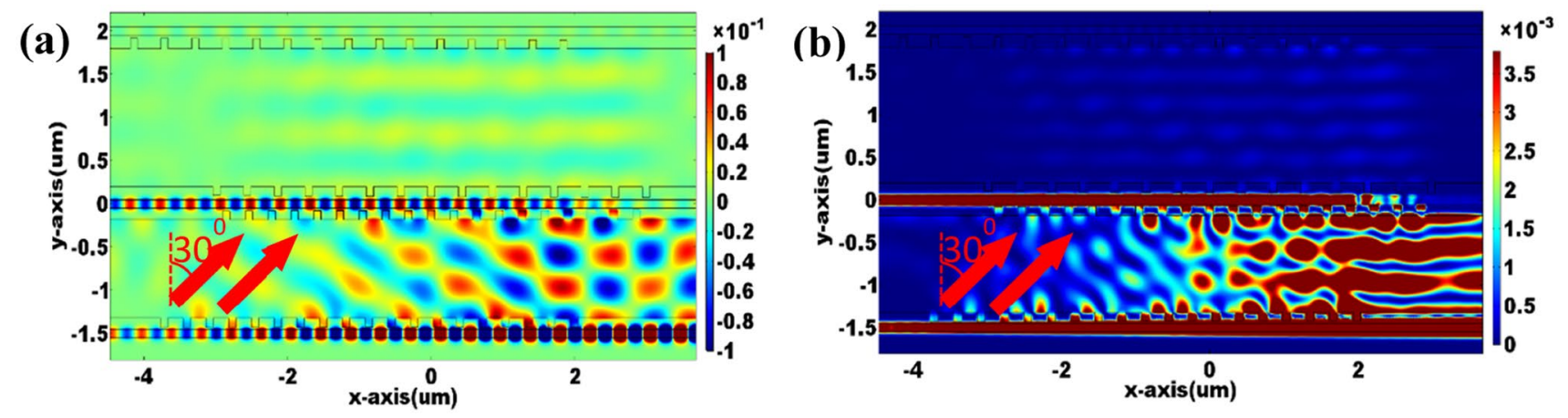

Fig. 8 Magnetic field $\left(H_{z}\right)$ in (a) and its intensity $\left(\left|H_{z}\right|^{2}\right)$ in $(\mathbf{b})$ for the proposed multiplexer with 650 nm wavelength for case 1 
nano-groove-array should be such that $\boldsymbol{k}_{\mathbf{0}}$ defines an angle $\theta$ to the right with respect to the normal to the bus.

Before we discuss the results further, it is to be mentioned that theoretically ER must be infinite with zero power flow in the undesired direction. In the practical realization, however, with a limited array size, interferences will not be as strong as expected ideally. Further, due to scattering of the incident wave on the nano-groove array at the coupling waveguide, additional undesired phase shifts may be introduced which will affect the structure's functionality $[11,13]$. This will result in some finite amount of power propagating in the opposite direction, leading to a finite ER. Having said that, an ER of $\geq 10 \mathrm{~dB}$ is reported to be good enough for practical applications [13] and the ER of all the proposed coupling structures discussed above are greater than $11 \mathrm{~dB}$ confirming the utility of our structures.

For the simulation of the $1 \times 2$ multiplexer/demultiplexer, we chose two wavelengths $\lambda_{1}=650 \mathrm{~nm}$ and $\lambda_{2}=850 \mathrm{~nm}$. The direction of $\boldsymbol{k}_{\mathbf{0}}$ is such that $\theta_{1}$ as well as $\theta_{2}$ is set as $30^{\circ}$ (Fig. 2). For the structure to function as per the design,
$650 \mathrm{~nm}$ should be coupled to the waveguide at the bottom and $850 \mathrm{~nm}$ should be coupled to the waveguide at the top. All the parameters remain the same as in the case $1 \mathrm{cou}-$ pler discussed earlier for $650 \mathrm{~nm}$ wavelength, whereas for $850 \mathrm{~nm}$, the parameters are as follows. Nano-groove spacing is $427 \mathrm{~nm}$ with 15 grooves within the $6 \mu \mathrm{m}$ size of the nanogroove-array; metal-barrier thickness is decided by (2) with $A=50 \mathrm{~nm}$ and $a=110 \times 10^{3}$ obtained by trial and error. The pickup waveguide was placed $2 \mu \mathrm{m}$ above the bus.

Figure $6 \mathrm{a}, \mathrm{b}$ show the magnetic field $\left(H_{\mathrm{Z}}\right)$ and its intensity $\left(\left|H_{\mathrm{Z}}\right|^{2}\right)$, respectively, for a case where the bus carries SPP waves at $650 \mathrm{~nm}$. Similarly, Fig. $6 \mathrm{c}$, d show the magnetic field $\left(H_{Z}\right)$ and its intensity $\left(\left|H_{Z}\right|^{2}\right)$, respectively, for a case where the bus carries SPP waves at $850 \mathrm{~nm}$. These figures in combination clearly show that the proposed structure can separate the two wavelengths successfully and also couple them to respective waveguides. The structure also ensures that propagation of coupled SPP waves are only in the desired direction with negligible flow of energy in
Fig. 9 Illustration of multiple functionalities integrated on an MIM bus; power splitter followed by a demultiplexer. (a) Structure, (b) magnetic field $\left(H_{z}\right),(\mathbf{c})$ magnetic field intensity $\left(\left|H_{z}\right|^{2}\right)$ for a wavelength of $650 \mathrm{~nm}$ (a)

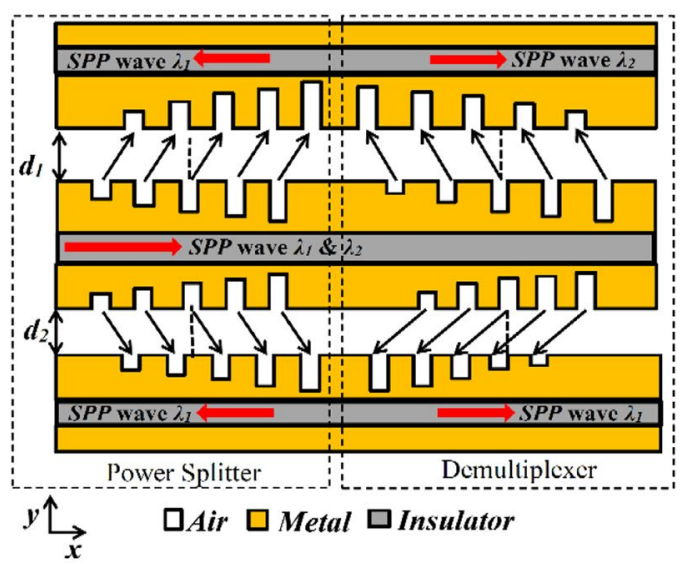

(b)

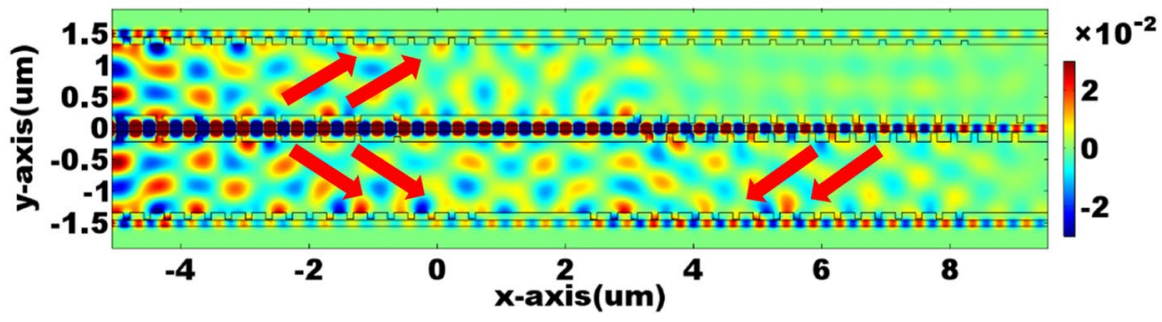

(c)

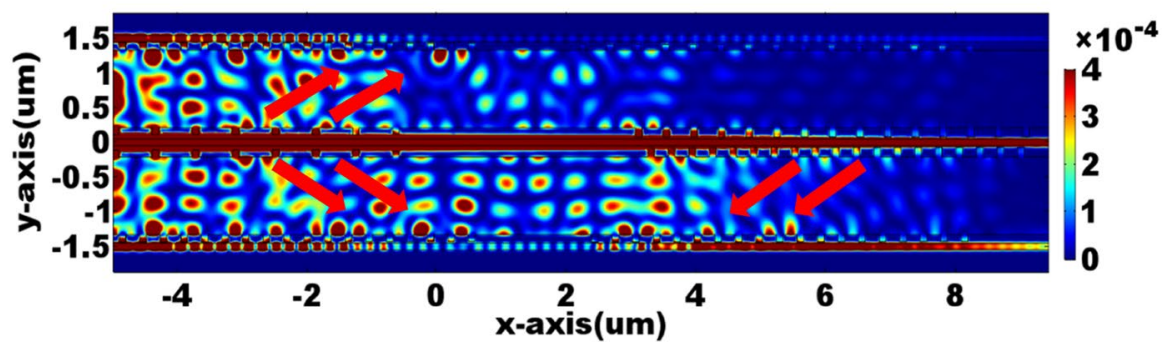


the opposite directions. The ER was found to be $16 \mathrm{~dB}$ for $650 \mathrm{~nm}$ and $19 \mathrm{~dB}$ for $850 \mathrm{~nm}$ wavelengths.

To evaluate the wavelength selectivity as well as bandwidth, the time averaged normalized magnitude of $x$ component of the power $\left(\left|P_{\mathrm{x}}\right|\right)$ at ports $P_{1}$ and $P_{2}$ (Fig. 2) was calculated for wavelengths ranging from 550 to $980 \mathrm{~nm}$. A plot of this can be found in Fig. 7. The bandwidth in terms of full width at half maximum (FWHM) was found to be $102 \mathrm{~nm}$ for the $650 \mathrm{~nm}$ wavelength and $71 \mathrm{~nm}$ for the $850 \mathrm{~nm}$ wavelength. The ability of the structure to reject the undesired wavelength can be clearly seen from this figure. An important figure of merit, the crosstalk, measures the amount of interference at the selected wavelength band from the rejected wavelength. In our case, the crosstalk at $650 \mathrm{~nm}$, measured as the ratio between powers at $P_{1}$ for $850 \mathrm{~nm}$ and $650 \mathrm{~nm}$, was found to be $-19 \mathrm{~dB}$. Through a similar calculation, the crosstalk at $850 \mathrm{~nm}$ was found to be $-18 \mathrm{~dB}$.

The demultiplexing structure discussed above will also act as a multiplexer due to the reciprocity theorem. For demonstration of multiplexer, we have simulated a case of $650 \mathrm{~nm}$ wavelength. To avoid unnecessary duplication, we have not included results for $850 \mathrm{~nm}$. All the parameters of the above demultiplexer were retained the same. The simulation results in terms of plot of magnetic field and its intensity can be found in Fig. 8. It can be clearly seen that the SPP wave of wavelength $650 \mathrm{~nm}$ from the bottom waveguide gets coupled into the bus as expected and propagates in the desired direction.

Another attractive characteristic of the proposed structures is that since these structures are etched in parallel to the MIM waveguide core, several such functionalities can be arranged sequentially on a strip of MIM waveguide to realize various fundamental signal processing operations. Figure 9a illustrates this important characteristic of these structures with its simulation result in Fig. 9b. It is to be noted that Fig. 9 illustrates only one out of several possible combinations. The SPP in the bus waveguide is of $650 \mathrm{~nm}$ wavelength. It first encounters a splitter which divides the power almost equally and couples it to the two waveguides at the top and bottom of the bus waveguide. Later, it encounters a demultiplexer which drops the $650 \mathrm{~nm}$ wavelength and couples it to the waveguide at the bottom in the desired direction.

\section{Conclusion}

An MIM unidirectional coupler that can couple SPP waves from one MIM waveguide to another and its extension to realize a $1 \times 2$ wavelength multiplexer/demultiplexer was proposed and simulated using finite element method technique. The design is based on the principle of interference and Helmholtz reciprocity theorem. The structure is realized with a nano-groove-array with the groove spacing adjusted for the desired interference effect. Simulation results presented confirm the functioning of these structures as per the design specifications. For the coupler, ER was found to be $>11 \mathrm{~dB}$ for all the cases discussed for an operating wavelength of $650 \mathrm{~nm}$. This confirms its utility in optical signal processing systems. The $1 \times 2$ multiplexer/ demultiplexer was simulated for wavelengths of $650 \mathrm{~nm}$ and $850 \mathrm{~nm}$. Results obtained gave a crosstalk of $-19 \mathrm{~dB}$ and FWHM of $102 \mathrm{~nm}$ for a wavelength of $650 \mathrm{~nm}$. These parameters were found to be $-18 \mathrm{~dB}$ and $71 \mathrm{~nm}$, respectively, for a wavelength of $850 \mathrm{~nm}$. More interestingly, it was shown that the method proposed in this paper has an inherent feature which enables integration of multiple functionalities on a single MIM waveguide. With these figures of merit and the capability of integrating multiple functionalities, the structures proposed in this paper are expected to have a wide range of applications in future nano-photonic signal processing systems.

Author Contribution Theoretical ideas were developed by both the authors. Simulations were carried out by Dr. Aparna Udupi and Dr. Sathish Kumar Madhava prepared the manuscript.

Funding Open access funding provided by Manipal Academy of Higher Education, Manipal

Data Availability The datasets generated during and/or analyzed during the current study are available from the corresponding author on reasonable request.

Code Availability The code used during the current study is available from the corresponding author on reasonable request.

\section{Declarations}

Conflict of Interests The authors declare no competing interests.

Open Access This article is licensed under a Creative Commons Attribution 4.0 International License, which permits use, sharing, adaptation, distribution and reproduction in any medium or format, as long as you give appropriate credit to the original author(s) and the source, provide a link to the Creative Commons licence, and indicate if changes were made. The images or other third party material in this article are included in the article's Creative Commons licence, unless indicated otherwise in a credit line to the material. If material is not included in the article's Creative Commons licence and your intended use is not permitted by statutory regulation or exceeds the permitted use, you will need to obtain permission directly from the copyright holder. To view a copy of this licence, visit http://creativecommons.org/licenses/by/4.0/. 


\section{References}

1. Maier SA (2007) Plasmonics: fundamentals and applications. Springer Science \& Business Media

2. Ozbay E (2006) Plasmonics: merging photonics and electronics at nanoscale dimensions. Science 311:189-193

3. Bozhevolnyi SI, Genet C, \& Ebbesen TW (2008) Surface-plasmon circuitry. Phys Today $44-50$

4. Yang R, \& Lu Z (2012) Subwavelength plasmonic waveguides and plasmonic materials. International Journal of Optics, 2012

5. Kazanskiy NL, Khonina SN, Butt MA (2020) Plasmonic sensors based on Metal-insulator-metal waveguides for refractive index sensing applications: a brief review. Phys E 117:113798

6. Abdulnabi SH, Abbas MN (2019) All-optical logic gates based on nanoring insulator-metal-insulator plasmonic waveguides at optical communications band. J Nanophotonics 13:016009

7. Hu F, Yi H, Zhou Z (2011) Band-pass plasmonic slot filter with band selection and spectrally splitting capabilities. Opt Express 19:4848-4855

8. Fu Y, Zhou X (2010) Plasmonic lenses: a review. Plasmonics 5:287-310

9. Gao Y, Liu J, Guo K, Gao Y, Liu S (2014) A side-illuminated plasmonic planar lens. Opt Express 22:699-706

10. Chen J, Li Z, Zhang X, Xiao J, Gong Q (2013) Submicron bidirectional all-optical plasmonic switches. Sci Rep 3:1451

11. Liu D, Li K, Lu F, Ho CC, Xu A (2014) Compact plasmonic unidirectional coupler with high performance based on an asymmetric slit-groove structure. J Lightwave Technol 32:4837-4843

12. Dionne JA, Lezec HJ, Atwater HA (2006) Highly confined photon transport in subwavelength metallic slot waveguides. Nano Lett 6:1928-1932

13. Liu D, Li K, Ho CC, Xu A, Li H (2015) Efficiently unidirectional coupling of surface plasmons based on asymmetric cascaded nanoslits. IEEE Photonics Technol Lett 27:1671-1674

14. Liang X, Wang J, Tang B, Xia X, Song C, Qu S, Liu C (2016) Unidirectional launcher of surface plasmon polaritons based on subwavelength slits with side-illumination and backsideillumination. Optik 127:1139-1143

15. Lu F, Wang Z, Tian Z, Xu A (2016) An efficient and ultra-broadband unidirectional optical coupler for wide incidence angles. Opt Commun 379:1-5

16. Wu YD (2014) High transmission efficiency wavelength division multiplexer based on metal-insulator-metal plasmonic waveguides. J Lightwave Technol 32:4844-4848

17. Hu F, Yi H, Zhou Z (2011) Wavelength demultiplexing structure based on arrayed plasmonic slot cavities. Opt Lett 36:1500-1502
18. Wang G, Lu H, Liu X, Mao D, Duan L (2011) Tunable multichannel wavelength demultiplexer based on MIM plasmonic nanodisk resonators at telecommunication regime. Opt Express 19:3513-3518

19. Xie YY, He C, Li JC, Song TT, Zhang ZD, Mao QR (2016) Theoretical investigation of a plasmonic demultiplexer in MIM waveguide crossing with multiple side-coupled hexagonal resonators. IEEE Photonics J 8:1-12

20. Chang W, Lu L, Ren X, Li D, Pan Z, Cheng M, Zhang M (2018) Ultra-compact mode (de) multiplexer based on subwavelength asymmetric Y-junction. Opt Express 26:8162-8170

21. Sumimura A, Ota M, Nakayama K, Ito M, Ishii Y, Fukuda M (2016) Low-return-loss plasmonic multiplexer with tapered structure. IEEE Photonics Technol Lett 28:2419-2422

22. Xu Y, Gao X, Bai D, Zhu G, Yuan J, Zhu H, Wang Y (2017) Free-space-to-waveguide demultiplexer featuring zero-contrast gratings. Optics Communications 387:89-94

23. Wang B, Wu X, Zhang Y (2013) Multiple-wavelength focusing and demultiplexing plasmonic lens based on asymmetric nanoslit arrays. Plasmonics 8:1535-1541

24. Kumar MS, Piao X, Koo S, Yu S, Park N (2010) Out of plane mode conversion and manipulation of surface plasmon polariton waves. Opt Express 18:8800-8805

25. Li L, Li T, Wang S, Zhu S, Zhang X (2011) Broad band focusing and demultiplexing of in-plane propagating surface plasmons. Nano Lett 11:4357-4361

26. Aparna U, Mruthyunjaya HS, Sathish Kumar M (2018) Plasmonic wavelength demultiplexer with mode conversion capabilities. Plasmonics 13:511-517

27. Born M, Wolf E (2013) Principles of optics: electromagnetic theory of propagation, interference and diffraction of light. Elsevier

28. Udupi A, Handigod MS, Kumar SM (2018) Unidirectional and bidirectional coupling of surface plasmons based on a nonperiodic nano slit array. Photonics Letters of Poland 10:17-19

29. Vial A, Grimault AS, Macías D, Barchiesi D, De La Chapelle ML (2005) Improved analytical fit of gold dispersion: application to the modeling of extinction spectra with a finite-difference timedomain method. Phys Rev B 71:085416

Publisher's Note Springer Nature remains neutral with regard to jurisdictional claims in published maps and institutional affiliations. 